\title{
Estímulos Visuais e Produção Escrita de Escolares com e sem Queixas de Alterações na Escrita
}

\author{
Visual Stimuli and Written Production of Students with and without \\ Complaints of Writing Disorders
}

\author{
Maria Silvia Cárnio*, Juliana Ramos Casemiro, Karen Barros Ribeiro \\ \& Aparecido José Couto Soares \\ Universidade de São Paulo, São Paulo, São Paulo, Brasil
}

\begin{abstract}
Resumo
O presente estudo objetivou verificar a influência de estímulos visuais na produção escrita de escolares com e sem queixas de alterações na escrita. Para tanto, 50 escolares de $4^{\circ}$ e $5^{\circ}$ anos, divididos em G1 (sem queixas) e G2 (com queixas) foram instruídos a realizar produções escritas com base em dois diferentes estímulos visuais. Utilizou-se para análise dos dados as Competências Comunicativas, sendo que em relação à Genérica, predominou a narração para ambos os estímulos, nos dois grupos. Quanto à Competência Enciclopédica, somente para as figuras em sequência, houve diferença significante. No que diz respeito à Competência Linguística, houve diferença apenas para a Coesão Global. Não houve influência dos estímulos visuais para ambos os grupos. Entretanto, fatores individuais e sociais podem ter influenciado os resultados obtidos.

Palavras-chave: Educação, escrita manual, avaliação, escolaridade, narração.

Abstract

This study aimed to verify the influence of visual stimuli on the written production of students with and without complaints of writing disorders. As subjects, it had 50 students from $4^{\text {th }}$ and $5^{\text {th }}$ grades of elementary school divided in G1 (with no complaints) and G2 (with complaints) who were instructed to perform a written production based on two different visual stimuli. Communicative Competence criteria were used in order to analyze the data. Regarding Generic Competence, the narrative genre was the most used for both groups. In relation to Encyclopedic Competence, there was significant difference only for the figures in sequence. Concerning Linguistic Competence, there was difference only in the Global Cohesion. There was no influence of the visual stimuli on the written production for both groups. Nevertheless, individual and social factors might have influenced the results.

Keywords: Education, handwriting, evaluation, schooling, narration.
\end{abstract}

Para escrever bem o indivíduo deve ser hábil em transformar as estruturas orais em estruturas escritas, dando forma ao texto (Myhill, 2009). Uma das maneiras de se produzir a escrita está na composição de narrativas, presentes desde cedo na infância em diversas perspectivas, seja escrita ou oral, lidas ou contadas (Özyildirim, 2009), em diferentes contextos, cultura e épocas.

No Brasil, quase a totalidade das crianças ingressam no sistema educacional (Fernandes, 2007). Contudo, não

\footnotetext{
* Endereço para correspondência: Rua Cipotânea, 51, Cidade Universitária, São Paulo, SP, Brasil 05360-160. E-mail: mscarnio@usp.br.

Auxílio à Pesquisa: Este trabalho foi financiado pela Fundação de Amparo à Pesquisa do Estado de São Paulo (Fapesp), sob protocolos (nº 2008/11474-5 e 2008/11478-0). Agradecimentos: À Equipe da Escola e aos sujeitos da pesquisa.

Trabalho realizado no Laboratório de Leitura e Escrita do Departamento de Fisioterapia, Fonoaudiologia e Terapia Ocupacional da Faculdade de Medicina da Universidade de São Paulo, São Paulo, SP, Brasil.
}

se pode negar que há uma parcela significativa da população brasileira com dificuldades referentes à apropriação e ao uso da linguagem escrita, sejam essas produzidas por fatores sociais, escolares ou familiares (Garcia, 2004). O fato é que esses indivíduos podem estabelecer uma relação de sofrimento, resistência e desprazer com a linguagem escrita, favorecendo o desconhecimento acerca de suas funções, valores e usos (M. L. C. A. Machado, 2007; Massi $\&$ Berberian, 2005).

Sabe-se que a elaboração e a implementação do Currículo Nacional por si só não é suficiente para mudar o quadro de indisciplina, formação docente precária, desinteresse pelo trabalho escolar e falta de relevância da aprendizagem para a vida social que principalmente a escola pública apresenta. Nesse sentido, uma série de transformações curriculares deve ser acompanhada de políticas voltadas para a formação continuada de professores, com salário digno, estrutura de apoio (acervo de livros, supervisão, materiais didáticos, instalações adequadas) e a valorização do trabalho docente (Moura \& Fonseca, 2003). 
Em virtude disso, há uma constante preocupação sobre como irá se desenvolver o processo de alfabetização. Para auxiliar os estudantes é importante compreender a origem de suas dificuldades e resolvê-las. Para tanto, é necessário o conhecimento de fatores que determinam o aprendizado da linguagem escrita (Santos, 2007). De acordo com Maingueneau (2002), existem três Competências que intervém no conhecimento do discurso e devem estar relacionadas para que o sujeito seja capaz de produzir um discurso adequado. A Competência Genérica é a capacidade de produzir enunciados no âmbito de certo número de gêneros. A Competência Linguística relaciona-se com o domínio da língua e a Competência Enciclopédica refere-se ao conhecimento de mundo.

A possibilidade de mensurar e qualificar as características das produções escritas auxilia a entender a aquisição e o desenvolvimento da linguagem oral e, em consequência, do aprendizado da escrita em crianças que apresentam alterações nesse processo (Miilher \& Ávila, 2006), uma vez que a avaliação da linguagem escrita é um índice sensível para detectar problemas sutis e residuais da linguagem oral (Bishop \& Clarkson, 2003).

Segundo Cárnio e Santos (2005), a aquisição da leitura e da escrita torna-se interessante para a criança à medida que esta entra em contato com situações sociais informais que permitam a diferenciação do uso social da escrita, revelando sua importância e sua relação com a oralidade. Marcuschi (2003) afirma que o desenvolvimento da escrita pode ser acelerado com as práticas baseadas no letramento escrito

Silva e Spinillo (2000), em estudo com crianças de $1^{\mathrm{a}}$ à $4^{\mathrm{a}}$ séries, analisaram a influência de diferentes situações de produção escrita, como: produção livre, produção oral/escrita, produção a partir de sequência de gravuras e reprodução de uma história ouvida. Como resultado, as autoras encontraram que a gravura foi importante para o desenvolvimento da narrativa escrita, pois proporcionou histórias mais elaboradas, dado que a sequência das quatro gravuras apresentadas englobava um tema, uma cena, os personagens, uma meta e uma situação-problema que deveriam ser resolvidas pelos personagens.

A importância da imagem no desenvolvimento da linguagem também foi notada por autores como Luchesi e Reily (2007), muito embora esses tenham estudado o papel do desenho elaborado pela própria criança. Além disso, Bohn, Roehrig e Pressley (2004), em pesquisa realizada para avaliar a eficácia dos professores em sala de aula, apontaram que os docentes que mais valorizavam o lúdico nas atividades escolares obtiveram maior envolvimento dos alunos nas tarefas que exigiam um longo empenho acadêmico. Nesse sentido, é possível inferir que a imagem é uma ponte para o processo de alfabetização e para as práticas terapêuticas.

Dessa forma, ressalta-se a importância de investigar o desenvolvimento da linguagem escrita em indivíduos com e sem queixas de alterações na escrita, de modo a observar como diferentes tipos de imagens podem contribuir para o desenvolvimento da produção escrita. Sendo assim, este estudo teve por objetivo verificar a influência de estímulos visuais na produção escrita de escolares com e sem queixas de alterações na escrita.

\section{Método}

\section{Considerações Éticas}

Esta pesquisa foi desenvolvida no Laboratório de Investigação Fonoaudiológica em Leitura e Escrita do Departamento de Fonoaudiologia, Fisioterapia e Terapia Ocupacional da Faculdade de Medicina da Universidade de São Paulo (FMUSP) após aprovação pela Comissão de Ética para Análise de Projetos de Pesquisa (CAPPesq), da referida instituição sob o protocolos nº 1066/08 e 065/2008.

\section{Local}

Os dados foram coletados em uma Escola Pública Estadual, da zona oeste de São Paulo, que atende famílias de classe média-baixa.

\section{Participantes}

Participaram 50 escolares, de $3^{\mathrm{a}}$ e $4^{\mathrm{a}}$ séries $\left(4^{\circ}\right.$ e $5^{\circ}$ anos) do Ensino Fundamental, divididos em dois grupos: O Grupo 1 (G1) foi formado por 25 sujeitos sem queixas de alterações na escrita; o Grupo 2 (G2) contou com 25 sujeitos com queixas de alterações na escrita. Os escolares foram selecionados, independente do gênero e faixa etária.

Para a seleção da amostra, adotaram-se como critérios de inclusão: respostas a 20dBNA nas frequências de $500 \mathrm{~Hz}$ a $4000 \mathrm{~Hz}$; frequentar a $3^{\mathrm{a}}$ ou $4^{\mathrm{a}}$ séries $\left(4^{\mathrm{o}}\right.$ e $5^{\mathrm{o}}$ anos $)$ do Ensino Fundamental da referida escola por dois ou mais anos, com a finalidade de se garantir a adaptação ao processo pedagógico da mesma; possuir nível alfabético e/ ou ortográfico (Ferreiro \& Teberosky, 1985) de escrita e ausência de queixas de alterações de linguagem oral e escrita. Esses últimos critérios foram avaliados por meio do Protocolo de Avaliação da Leitura e Escrita ${ }^{1}$, Questionário Informativo preenchido pelos professores (Soares, Jacinto e Cárnio, 2012) e anamneses realizadas com os pais. É importante ressaltar que o termo "queixas" se refere às dificuldades que o escolar apresentava, não acompanhando o desempenho acadêmico dos demais estudantes em sala de aula, mesmo com todo o acesso ao conteúdo curricular.

Os pais e/ou responsáveis que concordaram com a participação do(s) filho(s) na pesquisa, assinaram o Termo de Consentimento Livre e Esclarecido (TCLE). Além disso, os professores responsáveis das séries supracitadas também assinaram um TCLE concordando em fornecer dados referentes ao desempenho dos escolares.

\footnotetext{
${ }^{1}$ Alves, D., \& Cárnio, M. S. (1999). Protocolos para avaliação de Leitura e Escrita. Manuscrito não publicado, Departamento de Fisioterapia, Fonoaudiologia e Terapia Ocupacional, Universidade de São Paulo, SP, Brasil.
} 
Procedimentos para Seleção dos Sujeitos. Para a seleção de sujeitos foi utilizado um protocolo de anamnese com os pais com a finalidade de se obter dados referentes a antecedentes pessoais e familiares. Os professores foram orientados a preencher um questionário informativo sobre o desempenho acadêmico e características comportamentais de cada escolar da amostra, a fim de pré-selecionar adequadamente os sujeitos da pesquisa. Para confirmação dos critérios inclusivos, foi realizada triagem fonoaudiológica no que diz respeito à linguagem oral e escrita.

Além disso, realizou-se otoscopia e triagem auditiva por meio dos seguintes instrumentos: otoscópio Mini HEINE 300 e Audiômetro Pediátrico (Interacoustics Pediatric Audiometer - PA5), para garantir o nível de acuidade auditiva proposto.

\section{Provas Experimentais}

Para a realização das produções escritas foi utilizada uma figura de ação e quatro figuras em sequência (Casemiro, Ribeiro, Matta, Soares, \& Cárnio, 2011).

As figuras em sequência foram selecionadas com base em um estudo realizado por Burman, Evans, Nunes e Bell (2008) e Burman, Nunes e Evans (2007), para validar um instrumento sensível a pequenos progressos da aquisição na escrita. Em relação à seleção da figura de ação, esta foi retirada de um livro de história amplamente utilizado na área de linguagem para promover a escrita e discurso narrativo em crianças (Mayer, 1969).

Os escolares realizaram individualmente suas produções escritas e, para tanto, foram alocados em grupos pequenos de forma a não se comunicarem durante as provas. A aplicação das provas com os estímulos foi realizada em dois dias. Os escolares receberam a seguinte instrução oral: "Você receberá uma figura e/ou série de figuras e produzirá um texto por escrito sobre esta figura".
As produções escritas foram analisadas qualitativa e quantitativamente segundo os critérios das Competências Comunicativas (Linguística, Genérica e Enciclopédica), organizadas a partir das idéias de Maingueneau (2002), adaptadas por Lima e Cárnio (2007) e publicadas por Casemiro et al., 2011. Tais competências influenciam o discurso escrito e devem estar relacionadas para que o sujeito seja capaz de produzir um texto coerente e coeso.

A Competência Genérica refere-se à capacidade de produzir diferentes gêneros textuais (Maingueneau, 2002). Para análise dos gêneros textuais utilizou-se a tipologia do discurso baseada nas ideias de Dolz, Noverraz e Schneuwly (2004) que classificou o discurso da seguinte forma: Narrativa (mimeses, nas quais, por meio da criação de intriga ocorre a estimulação da imaginação; possui uma estrutura composta de introdução, conflito e conclusão, além de caracterizar o desenvolvimento de personagens, bem como descrições de tempo e espaço); Relato (apresentação de experiências pessoais num dado período de tempo e espaço); Argumentação (uso de ideias ou argumentos para convencer/persuadir; inclui apoio, refutação e negociação de argumentos conforme posição do autor); Expositivo (possui a específica função de aprimorar o conhecimento; pode ser usado para informar ou ensinar diferentes procedimentos de áreas diversas) e Descritivo (pode estar relacionado com atividades físicas, psicológicas ou ambientais).

A Competência Linguística é definida como o domínio da linguagem, enquanto que a Competência Enciclopédica envolve o conhecimento de mundo adquirido por meio de experiências individuais (Maingueneau, 2002).

Quanto à análise quantitativa, cada item analisado, com exceção da tipologia do discurso, recebeu uma pontuação, conforme descrição e classificação propostos por Casemiro et al. (2011). A pontuação geral, de cada

Tabela 1

Desempenho em Competência Genérica dos Sujeitos com e sem Queixas de Alterações na Escrita

Figura de ação

\begin{tabular}{|c|c|c|c|c|c|c|c|}
\hline & & \multicolumn{4}{|c|}{ Grupos } & \multirow{2}{*}{\multicolumn{2}{|c|}{ Total }} \\
\hline & & \multicolumn{2}{|c|}{ G1 } & \multicolumn{2}{|c|}{ G2 } & & \\
\hline & & $n$ & $\%$ & $n$ & $\%$ & $n$ & $\%$ \\
\hline \multirow{3}{*}{ Ação } & Descrição & 0 & 0,0 & 5 & 20,0 & 5 & 10,0 \\
\hline & Narração & 25 & 100,0 & 19 & 76,0 & 44 & 88,0 \\
\hline & Relato & 0 & 0,0 & 1 & 4,0 & 1 & 2,0 \\
\hline Total & & 25 & 100,0 & 25 & 100,0 & 50 & 100,0 \\
\hline
\end{tabular}

Figura em sequência

\begin{tabular}{|c|c|c|c|c|c|c|c|}
\hline \multirow[t]{3}{*}{ (1) } & & \multicolumn{4}{|c|}{ Grupos } & \multirow{2}{*}{\multicolumn{2}{|c|}{ Total }} \\
\hline & & \multicolumn{2}{|c|}{ G1 } & \multicolumn{2}{|c|}{ G2 } & & \\
\hline & & $n$ & $\%$ & $n$ & $\%$ & $n$ & $\%$ \\
\hline \multirow{3}{*}{ Sequencia } & Descrição & 0 & 0,0 & 10 & 40,0 & 10 & 20,0 \\
\hline & Narração & 25 & 100,0 & 13 & 52,0 & 38 & 76,0 \\
\hline & Relato & 0 & 0,0 & 2 & 8,0 & 2 & 4,0 \\
\hline Total & & 25 & 100,0 & 25 & 100,0 & 50 & 100,0 \\
\hline
\end{tabular}


Cárnio, M. S., Casemiro, J. R., Ribeiro, K. B. \& Soares, A. J. C. (2013). Estímulos Visuais e Produção Escrita de Escolares com e sem Queixas de Alterações na Escrita.

sujeito em cada prova, poderia variar de zero (0) a vinte e dois (22) pontos.

Para se garantir a confiabilidade dos dados contou-se com a participação de cinco juízes com experiência e atuação na área de leitura e escrita, que analisaram individualmente e pontuaram todas as produções escritas, após treinamento específico.

\section{Análise Estatística}

Foram realizados testes estatísticos comparativos entre as produções escritas com base na figura de ação e nas figuras em sequência. Em alguns casos a distribuição dos resultados impediu a aplicação de testes estatísticos específicos. Para a análise estatística, utilizou-se o Teste de Fisher, com nível de significância de 5\%.

\section{Resultados}

Em relação à Competência Genérica, não foi possível a aplicação de testes estatísticos, para ambos os estímulos. No entanto, observou-se que o gênero narrativo foi predominante para ambos os estímulos, porém, foram obtidas algumas produções escritas descritivas para G2, principalmente para a figura em sequência (Tabela 1).

Embora os dois estímulos visuais tenham propiciado o desenvolvimento da narração, alguns sujeitos produziram narrativas com sinais de argumentação quando o estímulo visual foi a figura de ação, sugerindo que tal estímulo propicie uma melhor elaboração do discurso. No que concerne à marcação de narrador e personagem, observou-se que a maioria dos sujeitos fizeram essa marcação quando o

Tabela 2

Desempenho em Competência Enciclopédica dos Sujeitos com e sem Queixas de Alterações na Escrita

Figura de ação

\begin{tabular}{|c|c|c|c|c|c|c|c|c|c|}
\hline & & & \multicolumn{4}{|c|}{ Grupos } & \multirow{2}{*}{\multicolumn{2}{|c|}{ Total }} & \multirow{3}{*}{$\begin{array}{l}\text { Teste de Fisher } \\
\qquad(p)\end{array}$} \\
\hline & & & \multicolumn{2}{|c|}{ G1 } & \multicolumn{2}{|c|}{ G2 } & & & \\
\hline & & & $n$ & $\%$ & $n$ & $\%$ & $n$ & $\%$ & \\
\hline \multirow{5}{*}{ Uso de título } & \multirow{3}{*}{ Ação } & 0 & 17 & 68,0 & 20 & 80,0 & 37 & 74,0 & \multirow{5}{*}{0,519} \\
\hline & & 1 & 0 & 0,0 & 0 & 0,0 & 0 & 0,0 & \\
\hline & & 2 & 8 & 32,0 & 5 & 20,0 & 13 & 26,0 & \\
\hline & \multirow[t]{2}{*}{ Total } & & 25 & 100,0 & 25 & 100,0 & 50 & 100,0 & \\
\hline & & 0 & 17 & 68,0 & 15 & 60,0 & 32 & 64,0 & \\
\hline \multirow[t]{3}{*}{ Intertextualidade } & \multirow[t]{2}{*}{ Ação } & 1 & 5 & 20,0 & 6 & 24,0 & 11 & 22,0 & \multirow[t]{2}{*}{ Não aplicável } \\
\hline & & 2 & 3 & 12,0 & 4 & 16,0 & 7 & 14,0 & \\
\hline & \multirow[t]{2}{*}{ Total } & & 25 & 100,0 & 25 & 100,0 & 50 & 100,0 & \multirow{5}{*}{ Não aplicável } \\
\hline \multirow{4}{*}{ Uso de inferências } & & 0 & 2 & 8,0 & 5 & 20,0 & 7 & 14,0 & \\
\hline & \multirow[t]{2}{*}{ Ação } & 1 & 7 & 28,0 & 17 & 68,0 & 24 & 48,0 & \\
\hline & & 2 & 16 & 64,0 & 3 & 12,0 & 19 & 38,0 & \\
\hline & Total & & 25 & 100,0 & 25 & 100,0 & 50 & 100,0 & \\
\hline
\end{tabular}

Figura em sequência

\begin{tabular}{|c|c|c|c|c|c|c|c|c|c|}
\hline & & & \multicolumn{4}{|c|}{ Grupos } & \multirow{2}{*}{\multicolumn{2}{|c|}{ Total }} & \multirow{3}{*}{$\begin{array}{l}\text { Teste de Fisher } \\
\qquad(p)\end{array}$} \\
\hline & & & \multicolumn{2}{|c|}{ G1 } & \multicolumn{2}{|c|}{ G2 } & & & \\
\hline & & & $n$ & $\%$ & $n$ & $\%$ & $n$ & $\%$ & \\
\hline \multirow{5}{*}{ Uso de título } & \multirow{3}{*}{ Ação } & 0 & 18 & 72,0 & 25 & 100,0 & 43 & 86,0 & \multirow{5}{*}{$0,014 *$} \\
\hline & & 1 & 0 & 0,0 & 0 & 0,0 & 0 & 0,0 & \\
\hline & & 2 & 7 & 28,0 & 0 & 0,0 & 7 & 14,0 & \\
\hline & \multirow[t]{2}{*}{ Total } & & 25 & 100,0 & 25 & 100,0 & 50 & 100,0 & \\
\hline & & 0 & 25 & 100,0 & 18 & 72,0 & 43 & 86,0 & \\
\hline \multirow[t]{4}{*}{ Intertextualidade } & \multirow[t]{2}{*}{ Ação } & 1 & 0 & 0,0 & 5 & 20,0 & 5 & 10,0 & \multirow[t]{2}{*}{ Não aplicável } \\
\hline & & 2 & 0 & 0,0 & 2 & 8,0 & 2 & 4,0 & \\
\hline & \multirow[t]{2}{*}{ Total } & & 25 & 100,0 & 25 & 100,0 & 50 & 100,0 & \\
\hline & & 0 & 2 & 8,0 & 7 & 28,0 & 9 & 18,0 & \multirow{4}{*}{ Não aplicável } \\
\hline \multirow[t]{3}{*}{ Uso de inferências } & \multirow[t]{2}{*}{ Ação } & 1 & 3 & 12,0 & 13 & 52,0 & 16 & 32,0 & \\
\hline & & 2 & 20 & 80,0 & 5 & 20,0 & 25 & 50,0 & \\
\hline & Total & & 25 & 100,0 & 25 & 100,0 & 50 & 100,0 & \\
\hline
\end{tabular}


estímulo visual foi a figura de ação, tanto para G1 quanto para G2.

Quanto à Competência Enciclopédica, os testes estatísticos foram aplicados apenas para o item Uso de Título, sendo que somente para a figura em sequência houve diferença estatisticamente significativa $(p=0,014)$ na qual G1 apresentou maior número de produções escritas com este recurso (Tabela 2).
No que se refere à Competência Linguística, foi possível a aplicação de testes estatísticos apenas para o item Coesão Global, sendo que somente para a figura em sequência houve diferença estatisticamente significativa $(p=0,023)$ pois a maioria dos escolares do G1 fez uso adequado deste recurso (Tabela 3 ).

Tabela 3

Desempenho em Competência Linguística dos Sujeitos com e sem Queixas de Alterações na Escrita

Figura de ação

\begin{tabular}{|c|c|c|c|c|c|c|c|c|c|}
\hline & & & & & & & & & Teste de Fisher $(p)$ \\
\hline & & & & 1 & & 2 & & & \\
\hline & & & $n$ & $\%$ & $n$ & $\%$ & $n$ & $\%$ & \\
\hline & & 0 & 0 & 0,0 & 4 & 16,0 & 4 & 8,0 & \\
\hline Extensão do texto & Ação & 1 & 10 & 40,0 & 20 & 80,0 & 30 & 60,0 & Não aplicável \\
\hline & & 2 & 15 & 60,0 & 1 & 4,0 & 16 & 32,0 & \\
\hline & Total & & 25 & 100,0 & 25 & 100,0 & 50 & 100,0 & \\
\hline & & 0 & 0 & 0,0 & 8 & 32,0 & 8 & 16,0 & \\
\hline Pontuação & Ação & 1 & 23 & 92,0 & 16 & 64,0 & 39 & 78,0 & Não aplicável \\
\hline & & 2 & 2 & 8,0 & 1 & 4,0 & 3 & 6,0 & \\
\hline & Total & & 25 & 100,0 & 25 & 100,0 & 50 & 100,0 & \\
\hline & & 0 & 18 & 72,0 & 20 & 80,0 & 38 & 76,0 & \\
\hline Ortografia & Ação & 1 & 6 & 24,0 & 3 & 12,0 & 9 & 18,0 & Não aplicável \\
\hline & & 2 & 1 & 4,0 & 2 & 8,0 & 3 & 6,0 & \\
\hline & Total & & 25 & 100,0 & 25 & 100,0 & 50 & 100,0 & \\
\hline & & 0 & 0 & 0,0 & 0 & 0,0 & 0 & 0,0 & \\
\hline Coesão global & Ação & 1 & 11 & 44,0 & 13 & 52,0 & 24 & 48,0 & 0,777 \\
\hline & & 2 & 14 & 56,0 & 12 & 48,0 & 26 & 52,0 & \\
\hline & Total & & 25 & 100,0 & 25 & 100,0 & 50 & 100,0 & \\
\hline
\end{tabular}

Figura em sequência

\begin{tabular}{|c|c|c|c|c|c|c|c|c|c|}
\hline & & & & & & & & & Teste de Fisher $(n)$ \\
\hline & & & & 1 & & 2 & & & reste de risher $(p)$ \\
\hline & & & $n$ & $\%$ & $n$ & $\%$ & $n$ & $\%$ & \\
\hline & & 0 & 1 & 4,0 & 2 & 8,0 & 3 & 6,0 & \\
\hline Extensão do texto & Ação & 1 & 9 & 36,0 & 22 & 88,0 & 31 & 62,0 & Não aplicável \\
\hline & & 2 & 15 & 60,0 & 1 & 4,0 & 16 & 32,0 & \\
\hline & Total & & 25 & 100,0 & 25 & 100,0 & 50 & 100,0 & \\
\hline & & 0 & 1 & 4,0 & 8 & 32,0 & 9 & 18,0 & \\
\hline Pontuação & Ação & 1 & 21 & 84,0 & 17 & 68,0 & 38 & 76,0 & Não aplicável \\
\hline & & 2 & 3 & 12,0 & 0 & 0,0 & 3 & 6,0 & \\
\hline & Total & & 25 & 100,0 & 25 & 100,0 & 50 & 100,0 & \\
\hline & & 0 & 14 & 56,0 & 13 & 52,0 & 27 & 54,0 & \\
\hline Ortografia & Ação & 1 & 8 & 32,0 & 6 & 24,0 & 14 & 28,0 & Não aplicável \\
\hline & & 2 & 3 & 12,0 & 6 & 24,0 & 9 & 18,0 & \\
\hline & Total & & 25 & 100,0 & 25 & 100,0 & 50 & 100,0 & \\
\hline & & 0 & 0 & 0,0 & 0 & 0,0 & 0 & 0,0 & \\
\hline Coesão global & Ação & 1 & 9 & 36,0 & 18 & 72,0 & 27 & 54,0 & $0,023 *$ \\
\hline & & 2 & 16 & 64,0 & 7 & 28,0 & 23 & 46,0 & \\
\hline & Total & & 25 & 100,0 & 25 & 100,0 & 50 & 100,0 & \\
\hline
\end{tabular}


Cárnio, M. S., Casemiro, J. R., Ribeiro, K. B. \& Soares, A. J. C. (2013). Estímulos Visuais e Produção Escrita de Escolares com e sem Queixas de Alterações na Escrita.

\section{Discussão}

Entender o processo de aquisição da linguagem escrita, identificando recursos facilitadores pode ser uma importante ferramenta tanto em âmbito clínico quanto educacional (Casemiro et al., 2011). Sob essa perspectiva, este estudo objetivou investigar a influência de diferentes estímulos visuais na produção escrita de escolares com e sem queixas de alterações na escrita.

Em relação à Competência Genérica, constatou-se que o gênero narrativo foi predominante para ambos os estímulos, para G1 e G2. Tal fato pode ter ocorrido em razão de as figuras em sequência propiciarem não apenas a descrição e identificação de ações, mas também, o surgimento de habilidades mais elaboradas, típicas da tipologia do discurso Narrativo (Bliss, McCabe, \& Miranda, 1998). Entretanto, foram obtidas maior número de produções escritas descritivas para o G2, principalmente para as figuras em sequência.

Em relação aos estímulos, observa-se que a figura de ação propiciou resultados ligeiramente superiores, para ambos os grupos, sugerindo que este tipo de estímulo pode ser importante para desencadear o gênero narrativo, que exige maior demanda de aspectos linguístico-cognitivos (Olive, Favart, Beauvais, \& Beauvais, 2009).

No que diz respeito à Competência Enciclopédica, a maioria dos sujeitos de ambos os grupos não fez uso de Título, dado que sugere imaturidade dos mesmos enquanto escritores, uma vez que o uso de título é um dos indicadores de domínio da escrita, pois conduz o leitor de forma antecipada a intuir o conteúdo da produção escrita (Ferreira \& Correa, 2008).

Os resultados também apontam para o fato de que a maioria dos sujeitos do G1 utilizou inferências adequadas, enquanto os sujeitos do G2 fez tal uso de forma parcialmente adequada, fato que pode ser explicado pela presença de elementos figurativos, ou seja, a linguagem visual representa a intenção e a mensagem que o autor quer transmitir, permitindo ao escritor realizar inúmeras inferências e interpretações (Paolucci, 2009). Em relação à Intertextualidade, muitos sujeitos, de ambos os grupos, não pontuaram para ambos os estímulos, demonstrando que possuem pouco conhecimento de outras produções literárias, já que a intertextualidade envolve a leitura de outros textos ou do conhecimento de outras histórias (Miranda, Simeão, \& Mueller, 2007).

A análise da Competência Linguística está intimamente interligada à Competência Genérica, uma vez que a estrutura narrativa engloba a organização de idéias dentro de uma sequência lógico-temporal, a qual é demarcada pelos sinais de pontuação ( $\mathrm{Ji}, 2008)$. Assim sendo, verificou-se que um número ligeiramente maior de escolares do G1 produziu textos longos para ambas as figuras, enquanto que a maioria dos escolares do G2 apresentou textos de extensão média, tanto para figura de ação quanto para as figuras em sequências.
Sabe-se que o texto escrito é convencionalmente apresentado em forma de parágrafos, sendo estes, fronteiras que facilitam o processo de leitura e apresentação da informação (Fávero, Andrade, \& Aquino 1999; Hinds, 1980; Hofmann, 1989). Somando-se a isto, as divisões dos parágrafos apresentam a descontinuidade temática, podendo ser: temporal, espacial, figura dramática, tópico e orientação (Ji, 2008).

Quanto à Pontuação, observou-se que, apesar do G1 ter apresentado um desempenho superior ao G2, o uso deste recurso foi deficitário para ambos os grupos, para os dois estímulos apresentados. Este dado corrobora os achados da literatura, nos quais diferentes autores afirmaram que o uso de marcadores linguísticos se desenvolve quando a criança tem contato com outros materiais escritos e com o avanço da escolaridade (Zuanetti, Corrêa-Schnek, \& Manfredi, 2008). Tal fato pode ocorrer, pois o escritor iniciante escreve como se fala, e na fala a contextualização é dada pela interação simultânea entre o emissor e o receptor. Sendo assim, o papel da escrita não está bem estabelecido e pode gerar o risco de ambiguidade pela ausência ou inadequação de Pontuação (Arfé \& Perondi, 2008).

Em relação à análise da Ortografia, a maioria dos sujeitos, de ambos os grupos, apresentou mais do que cinco erros ortográficos, para os dois estímulos apresentados. Apesar disso, houve indícios de melhora na ortografia para a figura de ação em comparação à figura em sequência. Já na comparação entre os grupos pesquisados, não houve indícios de diferença entre os mesmos.

Sabe-se que escrever ortograficamente é uma tarefa difícil, uma vez que é necessário um aprendizado contínuo e progressivo, envolvendo processos metacognitivos e metagráficos (análise da grafia) até dominar as regras ortográficas. Estas Competências devem conferir ao aprendiz a capacidade de explicar o motivo da forma de escrever uma ou outra palavra, aprendendo não só o princípio alfabético (associação fonema-grafema), como também a norma ortográfica com suas regularidades e irregularidades (análises metagráficas e conhecimentos sintáticos; Dias \& Ávila, 2008). O uso correto da ortografia envolve o conhecimento gramatical e é influenciado pela exposição a materiais escritos e pelo avanço da escolaridade (Zuanetti et al., 2008).

Como era esperado para estudantes de Ensino Fundamental, os erros mais encontrados foram os relativos às letras que são concorrentes na escrita de palavras, os quais demandam conhecimento morfológico e linguístico que não estão relacionados com a fase inicial de alfabetização. Os erros de representações múltiplas são dificuldades encontradas em séries iniciais e podem ocorrer até a fase adulta (Zorzi, 1998).

O último aspecto analisado da Competência Linguística refere-se à Coesão Global, cujos resultados demonstraram que a maioria dos sujeitos de ambos os grupos soube utilizá-la de forma parcial ou total, para ambos os estímulos. Entretanto, para a figura em sequência, foi encontrada 
diferença estatisticamente significante entre os grupos pesquisados, uma vez que o G1 realizou maior número de produções escritas com coesão global adequada.

A coesão é uma propriedade que define um bom texto ao nível do discurso em oposição à sequência de frases (Connor, 1984). Talvez, esse seja um indicador de que os escolares pesquisados estejam iniciando um processo de organização do discurso escrito. Dessa forma, a habilidade de encadear os elementos coesivos correlaciona-se com a alta qualidade do texto escrito, uma vez que a ordem lógica dos eventos possibilita a coerência do discurso para o leitor (Fávero et al., 1999).

Cabe ressaltar ainda que outros fatores podem estar relacionados à semelhança das produções escritas de G1 e G2. Pode-se citar a má qualidade do ensino nas escolas públicas do país, registradas nos mais variados indicadores; as condições de infraestrutura, a formação de muitos profissionais, os alunos desinteressados e até mesmo as condições familiares e socioeconômicas (N. J. Machado, 2007).

Conforme observado, além de o desempenho dos escolares apresentar-se ruim na maioria das Competências analisadas, não houve diferença estatisticamente significante entre os grupos pesquisados para a maioria dos itens. Porém, houve indícios de que G1 apresentou produções escritas com melhor desempenho do que o G2 nas competências analisadas.

Uma possível explicação para a não interferência dos estímulos visuais na produção escrita dos escolares deste estudo pode estar relacionada ao conteúdo das figuras. Embora tenham sido tomados todos os cuidados necessários na seleção dos estímulos visuais, somente depois de iniciada a análise das produções escritas, notou-se que ambos continham elementos que poderiam ser disparadores de uma narrativa, sendo esta uma limitação deste estudo.

Considera-se que estudos como estes são fundamentais para que se obtenham padrões de normalidade sobre produções escritas de escolares de Ensino Fundamental, principalmente utilizando-se estímulos visuais para que se possa ter uma padronização das produções escritas. Contudo, sugere-se, que seja feito um estudo piloto para a seleção dos estímulos eliciadores das produções escritas, bem como comparação entre indivíduos de escolas publicas e particulares.

\section{Conclusão}

Não houve influência dos estímulos visuais nas produções escritas de ambos os grupos. Entretanto, o G1 apresentou desempenho ligeiramente superior em relação a $\mathrm{G} 2$, porém com resultados inferiores ao esperado para a faixa de idade e escolaridade, descritas tanto na literatura nacional quanto na internacional. Tal dado pode indicar que fatores externos podem ter alguma influência sobre o processo de aquisição da linguagem escrita.

\section{Referências}

Arfé, B., \& Perondi, I. (2008). Deaf and hearing students' referential strategies in writing: What referential cohesion tells us about deaf students' literacy development. First Language, 28(4), 355-375.

Bishop, D. V. M., \& Clarkson, B. (2003). Written language as a window into residual language deficits: A study of children with persistent and residual speech and language impairments. Cortex, 39(2), 215-237.

Bliss, L., McCabe, A., \& Miranda, A. E. (1998). Narrative Assement Profile: Discourse Analysis for School-age Children. Journal Communication Disorders, 31, 347-363.

Bohn, C. M., Roehrig, A. D., \& Pressley, M. (2004). The first days of school in the classroom of two more effective and for less effective primary-grades teachers. Element Scholl Journal, 104(4), 269-287.

Burman, D., Evans, D., Nunes, T., \& Bell, D. (2008). Assessing deaf children's writing in primary school: Grammar and story development. Deafness and Education International, 10(2), 93-110.

Burman, D., Nunes, T., \& Evans, D. (2007). Writing profiles of deaf children taught through British sign language. Deafness and Education International, 9(1), 2-23.

Cárnio, M. S., \& Santos, D. (2005). Evolução da consciência fonológica em alunos de ensino fundamental. Pró-Fono: Revista de Atualização Científica, 17(2), 195-200.

Casemiro, J. R., Ribeiro, K. B., Matta, T. R. G., Soares, A. J. C., \& Cárnio, M. S. (2011). Interferências de estímulos visuais na produção escrita de escolares ouvintes sem queixas de alterações na escrita. Revista da Sociedade Brasileira de Fonoaudiologia, 16(4), 396-404.

Connor, U. (1984). A study of cohesion and coherence in English as a second language students' writing. Research on Language \& Social Interaction, 17(3), 301-316.

Dias, R. S., \& Ávila, C. R. B. (2008). Uso e conhecimento ortográfico no transtorno especifico da leitura. Revista da Sociedade Brasileira de Fonoaudiologia, 13(4), 381-390.

Dolz, J., Noverraz, M., \& Schneuwly, B. (2004). Sequências didáticas para o oral e a escrita: Apresentação de um procedimento. In R. Rojo \& G. S. Cordeiro (Eds. \& Trads.), Gêneros Orais e Escritos na Escola (pp. 95-128). São Paulo, SP: Mercado de Letras.

Fávero, L. L., Andrade, M. L. C. C. O., \& Aquino, Z. G. O. (1999). Oralidade e escrita: Perspectivas para o ensino de lingua materna. São Paulo, SP: Cortez.

Fernandes, R. (2007). Índice de Desenvolvimento da Educação Básica (Ideb). Brasília, DF: Instituto Nacional de Estudos e Pesquisas Educacionais Anísio Teixeira.

Ferreira, S. P., \& Correa, J. (2008). A influência de diferentes contextos de intervenção na escrita de histórias por crianças. Estudos de Psicologia (Campinas), 25(4), 547-555.

Ferreiro, E., \& Teberosky, A. (1985). Psicogênese da Língua Escrita (D. M. Lichtenstein, L. Di Marco, \& M. Corso, Trads.). Porto Alegre, RS: Artes Médicas.

Garcia, A. L. M. (2004). Fonoaudiologia e letramento. In A. T. B. C. Dauden \& C. C. Mori-de Angelis (Eds.), Linguagem escrita: Tendências e reflexões sobre o trabalho fonoaudiológico (pp. 15-35). São Paulo, SP: Pancast.

Hinds, J. (1980). Japanese expository prose. Papers in Linguistics: International Journal of Human Communication, 13, 117-158. 
Cárnio, M. S., Casemiro, J. R., Ribeiro, K. B. \& Soares, A. J. C. (2013). Estímulos Visuais e Produção Escrita de Escolares com e sem Queixas de Alterações na Escrita.

Hofmann, T. R. (1989). Paragraphs and anaphora. Journal of Pragmatics, 13, 239-250.

Ji, S. (2008). What do paragraph divisions indicate in narrative texts? Journal of Pragmatics, 40, 1719-1730.

Lima, F. T., \& Cárnio, M. S. (2007). Análise da produção escrita de surdos do ensino superior. São Paulo. Adaptado de Romano-Soares $S$. Práticas de narrativas escritas em estudantes do ensino fundamental (Dissertação de mestrado, Faculdade de Educação, Universidade de São Paulo, SP, Brasil).

Luchesi, K. F., \& Reily, L. (2007). O papel do desenho na clínica fonoaudiológica: Profissionais falam de sua prática. Distúrbio de Comunicação, 19(1), 51-61.

Machado, M. L. C. A. (2007). Grupo de linguagem escrita: Uma proposta de intervenção fonoaudiológica (Dissertação de mestrado, Universidade Tuiuti do Paraná, Curitiba, PR, Brasil).

Machado, N. J. (2007). Qualidade da educação: Cinco lembretes e uma lembrança. Estudos Avançados, 21(61), 277-294.

Maingueneau, D. (2002). Análise de textos de comunicação. São Paulo, SP: Cortez.

Marcuschi, L. A. (2003). Da fala para a escrita: Atividades de retextualização. São Paulo, SP: Cortez.

Massi, G. A., \& Berberian, A. P. (2005). A clínica fonoaudiológica voltada aos chamados distúrbios de leitura e escrita: Uma abordagem constitutiva da linguagem. Revista da Sociedade Brasileira de Fonoaudiologia, 10(1), 43-52.

Mayer, M. (1969). Frog, where are you?. New York: Pinguin.

Miilher, L. P., \& Ávila, C. R. B. (2006). Variáveis lingüísticas e de narrativas no distúrbio de linguagem oral e escrita. Pró-Fono: Revista de Atualização Científica, 18(2), 177-187.

Miranda, A., Simeão, E., \& Mueller, S. (2007). Autoria coletiva, autoria ontológica e intertextualidade: Aspectos conceituais e tecnológicos. Ciência da Informação, 36(2), 35-45.

Moura, M. C., \& Fonseca, S. G. (2003). Saberes e práticas de ensino de História: A implementação dos PCs nas séries iniciais do ensino fundamental. Ensino em Re-Vista, 11(1), 7-25.

Myhill, D. (2009). From talking to writing: Linguistic development in writing. British Psychological Society, 1(1), 27-44.

Olive, T., Favart, M., Beauvais, C., \& Beauvais, L. (2009). Children's cognitive effort and fluency in writing: Effects of genre and of handwriting automatisation. Learning and Instruction, 19, 299 -308.

Özyildirim, I. (2009). Narrative analyses: An analysis of oral e written strategies in personal experience narratives. Journal of Pragmatics, 41, 1209-1222.

Paolucci, J. F. (2009). Estudo das correlações entre a função pragmática da linguagem e a produção textual escrita (Tese de doutorado, Universidade Federal de São Paulo, SP, Brasil).

Santos, M. T. M. (2007). Vocabulário, consciência fonológica e nomeação rápida: Contribuições para a ortografia e elaboração escrita (Tese de doutorado, Universidade de São Paulo, SP, Brasil).

Silva, M. E. L., \& Spinillo, A. G. (2000). A influência de diferentes situações de produção na escrita de histórias. Psicologia: Reflexão e Crítica, 13(3), 337-350.

Soares, A. J. C., Jacinto, L. A., Cárnio, M. S. Memória operacional fonológica e consciência fonológica em escolares ao final do ciclo I do ensino fundamental. Rev Soc Bras Fonoaudiol. 2012;17(4):447-53

Zorzi, J. L. (1998). Aprender a escrever - A apropriação do sistema ortográfico. Porto Alegre, RS: Artes Médicas.
Zuanetti, P. A., Corrêa-Schnek, A. P., \& Manfredi, A. K. S. (2008). Comparação dos erros ortográficos de alunos com desempenho inferior em escrita e alunos com desempenho médio nesta habilidade. Revista da Sociedade Brasileira de Fonoaudiologia, 13(3), 240-245. 